\title{
La traducción de criptogramas y poemas en El Código Da Vinci, de Dan Brown
}

\author{
Allan Pineda Rodríguez ${ }^{1}$ \\ Universidad Nacional, Costa Rica
}

\section{Resumen}

Se desarrolla un análisis comparativo entre los criptogramas y poemas originales de la novela de Dan Brown, The Da Vinci Code, y su correspondiente traducción al español, realizada por Juanjo Estrella. El trabajo de contraste analiza la traducción de cada uno de los casos y evalúa la labor del traductor, motivando a la vez a que los lectores sean más críticos en su práctica literaria.

\section{Abstract}

A comparative analysis is developed here between the original cryptograms and poems found in Dan Brown's novel, The Da Vinci Code, and its Spanish counterpart translated by Juanjo Estrella. This analysis focuses on the translation of each of the cases and the work done by the translator, thus encouraging readers to become more critical in their readings.

Palabras clave: Da Vinci, criptograma, anagrama, traducción, poema, poesía Keywords: Da Vinci, cryptogram, anagram, translation, poem, poetry

No es decir que la traducción literaria es uno de los trabajos más complejos que enfrentan los traductores. La traducción arroja sorpresas y

$\overline{l \quad C o r r e o ~ e l e c t r o ́ n i c o: ~ a p i n e d a r @ y a h o o . c o m . ~}$ 
retos que a veces son muy complicados, pero una vez "cabalgando bestia" lo que queda es domarla y cuando se logra queda la satisfacción de haber derribado los muros que se yerguen entre los idiomas.

Tal es el caso de la traducción literaria que en comparación con otros tipos de traducción, como la técnico-científica; exige algo más, que sólo el tipo de texto literario puede expresar pues transmite emociones, pasiones, misterios y muchos otros sentimientos; el traductor se encarga de enviar esos mismos sentimientos a la lengua meta. Como lo afirma Cristián Rodríguez, "las traducciones literarias demandan facultades que no se exigen a los traductores comerciales"? Por esto y mucho más la traducción literaria ostenta un lugar privilegiado para algunos traductores, puesto que además de resultar lucrativa (sólo basta mencionar el fenómeno Harry Potter) también produce la satisfacción de que su obra será leída por millones de personas en todo el orbe.

Para ser traductor literario hay que contar con dotes de escritor; esto significa que para traducir esta clase de textos hay que dominar muchos aspectos de la composición literaria. Hay que saber utilizar toda clase de figuras literarias desde los más simples símiles, hasta las más complejas metáforas, pasando por el uso del hipérbaton, el epíteto y la perífrasis; y tener un amplio conocimiento de vocabulario, sin dejar de lado aspectos de la poesía y sus diferentes estructuras. O sea, todo eso que enriquece un escrito literario.

Todas estas formas literarias constituyen un problema por resolver para los traductores y dentro de ellas los mensajes cifrados no son la excepción. Los criptogramas o mensajes escritos con claves secretas, proponen su descodificación al ordenar palabras y letras, colocándolas según indicaciones o reglas previamente establecidas. Entre los criptogramas se encuentran los monogramas que pueden representar secretamente una o varias palabras que emplean sus iniciales y que comúnmente se usan como abreviaturas. Los anagramas

$\overline{2} \quad$ Cristián Rodríguez. "Traducciones comerciales y traducciones literarias”, Letras 36 (2004) 219. 
surgen cuando se hace una transposición de las letras de una palabra o frase, con el propósito de representar un mensaje en clave.

\section{La novela El Código Da Vinci}

The Da Vinci Code ${ }^{3}$ es la tercera novela del escritor estadounidense Dan Brown, quien en los últimos años por sus escritos se ha visto implicado en debates sobre ciencia y religión. Esta novela encierra otra historia un tanto diferente y no conocida acerca del legado de Jesús. En el aspecto literario, uno de los puntos interesantes es la forma astuta de transmitir mensajes por medio de claves escondidas y acertijos sagaces, que revelan poco a poco historias, mitos, pistas y misterios que le dan figura a la obra, lo cual es una técnica literaria ya empleado por grandes escritores como Edgar Allan Poe (El escarabajo de oro). Esta novela presenta entre sus diversas características literarias el uso obligatorio de los criptogramas como recurso de ficción, ya que se trata de una novela de misterio en la cual hay que descifrar mucho más que las obras y pensamientos del af amado artista e inventor Leonardo Da Vinci.

Dado el tipo de novela de ficción y con solo observar el título, la codificación de mensajes es un elemento trascendental, no solo por la trama de la historia, sino porque la novela relaciona la criptografía con la Iglesia Cristiana. Esto tiene su fundamento histórico porque se cree que desde la antigüedad los primeros cristianos empleaban códigos secretos para comunicarse y proteger, cuidar y ocultar sus más importantes secretos.

En la novela, los mensajes ocultos son propuestos por uno de los personajes, Jacques Saunière, reconocido conservador del Museo del Louvre. Él siente una obligación de transmitir sus conocimientos y verdades a su nieta, Sophie Neveu, agente del departamento de criptografía de la Dirección Central de la Policía Judicial Francesa. Sin

3 Dan Brow'n. The Da Vinci Code (Nueva York: Doubleday. 2003). 
embargo, para descifrar los mensajes necesitará de alguien con conocimientos histórico-religiosos; este último personaje es Robert Langdon, profesor de simbología religiosa de la Universidad de Harvard. Cuando Jacques Saunière es asesinado, Sophie y Robert inician la búsqueda de la verdad tras los mensajes dejados por Saunière. Esto los lleva por incontables aventuras y persecuciones, que llenan de emoción al lector, involucrándolo a tal punto de tratar de descifrar junto con ellos los mensajes ocultos del abuelo de Sophie.

La obra fue escrita en inglés y se encasilla en el género de ficción; fue publicada en 2003 por la editorial Doubleday. Se ha catalogado como un Best Seller por haberse vendido más de cuarenta millones de ejemplares. Esta novela tiene la característica de presentar además del inglés, textos en francés y latín además de otras lenguas antiguas, lo cual representa otro reto adicional al de los criptogramas y versos para presentar la obra en español.

Por su parte el texto meta — es decir la versión al español de $E l$ Código Da Vinci ${ }^{4}$ — es la traducción de Juanjo Estrella. Se desconoce si existen otras versiones al español, pero en el presente artículo se analizará la forma en que Estrella trata y enfrenta la mutación de estos criptogramas y poemas al idioma español. Esta versión es de la editorial Umbriel y se publicó el mismo año que la novela original, 2003.

\section{Análisis comparativo}

La trama de la novela gira en torno a una serie de pistas que los protagonistas deben seguir para descubrir un secreto. Tales pistas son mensajes escritos en clave o acertijos en forma de versos. La tarea del traductor consistió en trasladar esos mensajes secretos al español guardando el mismo sentido y sensación de misterio que tienen en la versión original, asunto que se revela con el estudio de cada ejemplo. La investigación que se presenta a continuación incluye precisamente esos mensajes en el mismo orden en que aparecen en la novela.

$4 \quad$ Dan Brown. El Código Da Vinci, trad. de Juanjo Estrella (Barcelona: Umbriel, 2003). 
En cuanto al análisis de los criptogramas se sigue el siguiente orden: presentación de los critptogramas, tanto la versión original como la de español, una descripción sintáctica de los elementos del criptograma en inglés, la misma descripción en español y finalmente el mensaje ya descifrado en ambos idiomas junto con el análisis del trabajo del traductor para causar el mismo efecto que en el texto original.

Para el análisis de los poemas se presenta el poema o estrofa original, seguidamente su análisis métrico y a continuación la traducción de su contenido, para tener una idea clara y general del mismo en español. Una vez hecho, se presenta la versión de Estrella y el análisis métrico junto con las consideraciones correspondientes en cuanto al trabajo de la traducción al español.

\section{Criptograma 1}

En el capítulo 8 está el primer criptograma formado por tres líneas. El primer renglón indica una serie de números y el segundo y tercero presentan dos frases.

Versión original:

$$
\text { 13-3-2-21-1-1-8-5 }
$$

O, Draconian devil!

Oh, lame saint! ${ }^{5}$

En este caso en la serie numérica no hay mayor cambio, tanto en inglés como en español esos números no tienen variación pues están colocados así debido a que son una secuencia específica la cual tendrá que descifrar los protagonistas. Los números no varían en la traducción ya que ese mismo desorden corresponde a la hora de querer descifrar su contenido tanto en inglés como en español.

$\overline{5}$ Brown. The Da Vinci Code. 47. 
En la segunda línea se encuentra la frase "O, Draconian devil". Tiene en inglés el siguiente análisis sintáctico:

- "O", interjección que se emplea para denotar sorpresa, dolor, etc.

- "Draconian", adjetivo que define características de "Draco", quien fue un legislador ateniense con fama de cruel y severo.

- "devil", sustantivo que comúnmente se conoce como el "diablo".

En la tercera línea la frase "Oh, lame saint" se analiza de la siguiente forma:

- "Oh", también interjección que se usa para expresar sorpresa, dolor, etc.

- "lame", adjetivo cuya definición más común se refiere a "cojo" o "renco".

- "saint", sustantivo que se interpreta como "santo".

Para el lector que aún no ha hecho su análisis criptográfico el mensaje tiene el sentido exclamativo de "Diablo draconiano" y "Santo renco". Sin embargo, el traductor ha de tener en cuenta que esto es un criptograma y que tiene un significado totalmente diferente al presentado en su lectura y forma natural.

La secuencia numérica no varía. La versión de la traducción al español debería tener el objetivo de transmitir el mismo mensaje escondido y causar el mismo efecto que el texto original.

Versión al español:

$$
\text { 13-3-2-21-1-1-8-5 }
$$

¡Diavole in Dracon!

Límala, asno ${ }^{6}$

Segunda línea, “¡Diavole in Dracon!”. De nuevo una frase exclamativa, solo que para sorpresa de los lectores esta se escribe en

$\overline{6}$ Brown. El Código Da Vinci, 61. 
italiano. Sin embargo, vale la pena resaltar la semejanza de significado de la frase.

- "Diavolo", sustantivo que proviene del italiano para "diablo".

- “in”, es la preposición para el español "en”.

- "Dracon", sustantivo que se relaciona al igual que la versión original al cruel legislador ateniense.

En la tercera línea la frase "Límala, asno" está en español y está conformada por sólo dos palabras a diferencia de la versión original que tiene tres.

- "Límala", en este caso es la conjugación imperativa del verbo "limar", o sea desgastar, pulir y hasta debilitar algo, no se dice qué.

- "Asno", se refiere al sustantivo que debe ejecutar la acción imperativa del verbo "limar"; el "asno" también es conocido como "burro", o podría referirse de manera coloquial a una persona de corto entendimiento, una persona "necia" o "torpe".

Una vez que el traductor tiene el mensaje descifrado debe pensar en cómo elaborar la versión en el idioma meta que contenga las claves necesarias para obtener el mismo resultado; es decir, descifrable de la misma manera. En este criptograma se emplean anagramas (mejor dicho, las letras están en desorden) y que hay que ordenarlas correctamente para descifrar lo que realmente quieren decir.

Para resolver esto Estrella no traduce las frases del inglés, puesto que aunque tuvieran sentido como " $\mathrm{O}$, Diablo draconiano!” y “ $\mathrm{OOh}$, santo renco!" serían inútiles para descifrarlos como anagramas debido a que dichas traducciones pierden algunas letras y agregan otras que no vienen al caso del mensaje descifrado.

Entonces, si se colocan las letras de ambos mensajes en el orden correcto, se obtiene el siguiente resultado: 


\begin{tabular}{|c|c|}
\hline Versión original & Mensa je descif rado (inglés) \\
\hline O, Draconian devil! & Leonardo Da Vinci! \\
Oh, lame saint! & The Mona Lisa! \\
\hline
\end{tabular}

\begin{tabular}{|c|c|}
\hline Versión al español & Mensa je descifrado (español) \\
\hline $\begin{array}{c}\text { ¡Diavole in Dracon! } \\
\text { ¡Límala, asno! }\end{array}$ & ¡Leonardo Da Vinci! \\
¡La Mona Lisa! \\
\hline
\end{tabular}

Como puede observarse en este ejemplo el truco radica en hacer primero una traducción del mensaje oculto y luego presentarlo con una frase que incluya las letras necesarias para que una vez al ordenarlo correctamente dé como resultado el mensaje final. El arte del traductor es escribir algo con cierto sentido en dicha frase, y que tenga similitud con la expresión original que también oculta el mensaje, pero también resolver el anagrama.

El proceso a seguir es el siguiente:

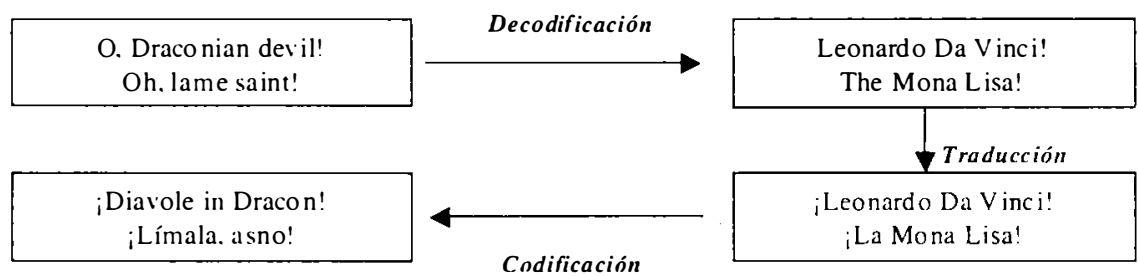

En la primera frase Estrella logra una buena traducción al italiano, que se asemeja al español, con un sentido casi perfecto al mensaje original en inglés y que sirve de igual forma, ya que esta frase la utiliza Robert Langdon para introducir ciertos conceptos sobre la Iglesia. Sin embargo, como en la otra frase no encontró traducción al español de "lame saint" que tuviera las mismas letras con un significado 
equivalente al original, no la puede utilizar. El traductor tiene que basar el diálogo del protagonista en la primera frase, estableciendo la relación con la Iglesia por mera deducción y no pudiendo utilizar al "santo renco" para referirse a la misma. Por eso opta por escribir una especie de orden ofensiva, que no tiene correspondencia con su versión en inglés y pierde importancia al relacionar las frases con la historia de la Iglesia, pero que logra su cometido al poder descifrarse como "La Mona Lisa".

Existe un error (que bien podría ser de imprenta): la primera vez que aparece el anagrama de "límala asno", no tiene símbolos de exclamación, a diferencia de la versión original que sí trae dicho signo. Pese a esto, cuando aparece más adelante en la novela, trae consigo los símbolos exclamativos ausentes en la primera ocasión.

\section{Criptograma 2}

En el capítulo 12 aparece una frase, continuación del primer criptograma, "P.S. Find Robert Langdon"7 . Está línea se tomará como ejemplo, porque se encuentra en otro capítulo y debido a que en este caso no se trata de una palabra sino de iniciales. Ahora lo importante es descifrar el significado de la abreviatura "P.S".

- $\quad$ "P.S.", para el lector original el significado es "Postscript", lo que se agrega a un escrito que ya ha sido concluido o firmado. En este caso se referiría a que existe información adicional al mensaje original y que esta sería que se buscara al señor Robert Langdon.

Hasta ahora no habría ningún problema, puesto que lo más lógico sería emplear su equivalente en español, "P.D." o "Post Data". No obstante, el mensaje tiene un significado especial ya que viene a ser otro criptograma, esta vez un monograma (representación compuesta

7 Brown. The Da Vinci Code, 74. 
por dos o más letras) en donde las iniciales "P.S." no necesariamente significan "Postscript" (o Post Scriptum). Así pues, el traductor tiene que emplear su astucia nuevamente e idear la forma de que esas mismas letras, que significan algo específico, permanezcan de igual forma en el texto traducido.

En la versión al español aparece de la siguiente forma: "P.S. Buscar a Robert Langdon"8. Ahora la pregunta es cómo defender el uso de "P.S." en lugar de "P.D.". Pues bien, Estrella cde nuevo acude a otro idioma que no sea el español; en este caso al latín para "P.S.": Post Scriptum, y que para efectos de la historia calza perfectamente. Al igual que "P.D.", "P.S." se referiría a un texto agregado al mensaje y de nueva cuenta funcionaría para descifrar que las iniciales "P.S." se refieren al francés de "Princesse Sophie", apelativo con el cual Jacques Saunière llamaba a su nieta Sophie Neveu. De igual forma las iniciales "P.S." se refieren más adelante en la novela de una u otra forma al "Prieuré de Sion" en francés, "Priory of Sion" en inglés y "Priorato de Sión" en español; otra razón por la cual en realidad esas iniciales no podrían haber cambiado.

\section{Criptograma 3}

En el capítulo 28 aparece otro anagrama:

Versión original:

\section{SO DARK THE CON OF MAN ${ }^{10}$}

Esta vez la frase original está compuesta por seis palabras. Este es otro mensaje secreto que Jacques Saunière deja a Sophie y Robert. He aquí el análisis sintáctico:

$8 \quad$ Brown, El Código Da Vinci, 91.

9 Priorato de Sión: una de las sociedades secretas más antiguas del mundo... guardianes de un antiguo secreto. Brown. El Código Da Vinci, 144.

10 Brown. The Da Vinci Code. 133. 
- " “SO", adverbio que se refiere a "tan" o "tanto", con el propósito de aumentar o exaltar algo.

- "DARK", adjetivo que se traduciría como "oscuro".

- “THE”, artículo definido, cuya interpretación dependerá del sustantivo al cual se refiera.

- "CON", sustantivo que se puede interpretar como "engaño", "mentira", "timo" o "fraude".

- "OF", preposición empleada para indicar posesión, conexión o asociación. Se puede entender en español como la preposición "de" y dicha asociación se daría con el siguiente sustantivo.

- "MAN", este último sustantivo es el que se relaciona con la anterior preposición y aunque esta palabra tiene un significado básicamente masculino, debería entenderse como "hombre" en el sentido de una denominación genérica refriéndose a la especie humana.

Así pues, si se desea saber el significado en español tendría esta traducción aproximada: "TAN OSCURO EL ENGAÑO DEL HOMBRE”, oración coherente desde el punto de vista semántico y, al igual que en la frase en inglés, se relacionaría con la novela cuando Robert Langdon relata la historia de que según los principios del Priorato de Sión, la Iglesia habría engañado al mundo.

Una vez comprendido el significado de la frase original, Estrella se enfrenta a un difícil trabajo. Necesita presentar una traducción con sentido similar y que además cumpla su función a la hora de descifrar el anagrama. A continuación se presenta el análisis de la versión al español.

Versión al español:

\section{NO VERDAD LACRA IGLESIAS ${ }^{11}$}

A diferencia de la versión original esta propuesta tiene sólo cuatro palabras en lugar de seis. Seguidamente se presenta el análisis de la misma.

- "NO", adverbio de negación.

11 Brown. El Código Da Vinci. 157. 
- "VERDAD", sustantivo, sinónimo de realidad, o sea hecho de existir realmente, antónimo de mentira.

- "LACRA", otro sustantivo, en este caso se refiere a un daño, defecto o vicio, mancha o falta. Aunque se puede interpretar tanto físico como moral, en este caso es estrictamente moral.

- "IGLESIAS", sustantivo que se refiere al estado eclesiástico que comprende a todos los ordenados; seguidores de las doctrinas religịosas. Aunque esté en plural se refiere a la Iglesia Católica, en especial a sus representantes.

Como resultado, el traductor propone una frase que a diferencia de su versión original es más directa al primero hablar de "no verdad" y luego expresar una idea despectiva de "lacra" y rematar con la palabra "iglesias" refiriéndose al Catolicismo, algo así como: "mentirosos, lacra de la iglesia". Es importante recalcar que esta traducción expresa un mensaje muchísimo más directo que en la versión en inglés.

Con esto queda resuelto el modo de expresar el mensaje. Ahora le toca el turno al anagrama y ver si funciona o no. A continuación se descifra el mensaje.

\begin{tabular}{|c|c|}
\hline Versión original & Mensaje descifrado (inglés) \\
\hline SO DARK THE CON OF MAN & MADONNA OF THE ROCKS \\
\hline
\end{tabular}

\begin{tabular}{|c|c|}
\hline Versión al español & Mensaje descifrado (español) \\
\hline NO VERDAD LACRA IGLESIAS & VE A LA VIRGEN DE LAS ROCAS* \\
\cline { 2 - 2 } & ID A LA VIRGEN DE LAS ROCAS \\
\hline
\end{tabular}

* De nueva cuenta se cometió un error en la versión al español, haya sido de edición o de impresión. El error aparece al tratar de completar el mensaje con las letras del anagrama y sobrar las letras "D", "I". Así pues. la traducción correcta debió ser "ID A LA VIRGEN ..." en lugar de "VE A LA VIRGEN...". 
El proceso para la traducción de este anagrama sería el siguiente:

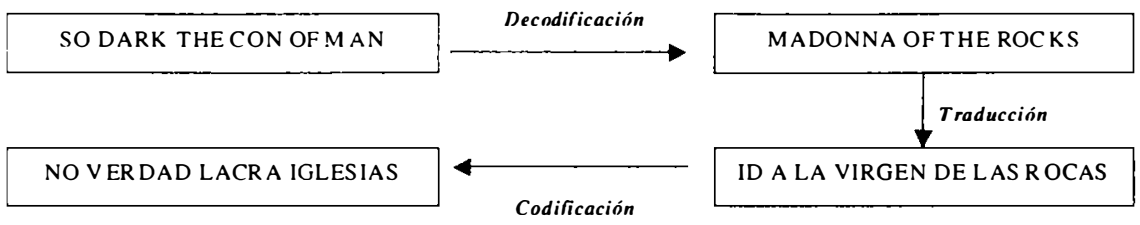

El traductor traslada con las palabras pertinentes, aunque totalmente diferentes al original pero con las letras necesarias, el mensaje escondido. Tal mensaje se refiere a otra de las pinturas de Da Vinci, "La virgen de las rocas" en donde los protagonistas encontrarían otra de las pistas en su aventurado recorrido.

\section{Poema 1}

En el capítulo 72 aparece el primer poema que es necesario descifrar para que los protagonistas continúen su travesía. Esta vez ya no es un criptograma, ahora no se trata de ordenar las palabras o letras, sino interpretar lo que les dice el poema y así obtener la palabra clave que abre el criptex ${ }^{12}$.

Versión original:

an ancient word of wisdom frees this scroll and helps us keep her scatter'd family whole

a headstone praised by templars is the key and atbash will reveal the truth to the $e^{13}$

Es una estrofa de cuatro versos con una métrica específica: el pentámetro yámbico: versos de cinco pies métricos que a su vez se

12 Criptex: especie de caja fuerte que sirve para guardar información secreta. Brown, El Código Da Vinci, 250.

13 Brown. The Da Vinci Code, 328. 
forman por una sílaba breve (átona) y otra larga (tónica) y que tiene rima en los dos primeros y en los dos últimos versos.

A continuación, el análisis métrico del poema original:

$\begin{array}{lllll}1 & 2 & 3 & 4 & 5\end{array}$

an an / cient word / of wis / dom frees / this scroll

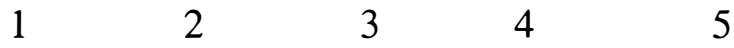

and helps / us keep / her scat / ter'd fam / ly whole

$\begin{array}{lllll}1 & 2 & 3 & 4 & 5\end{array}$

a head / stone praised / by tem / plars is / the key

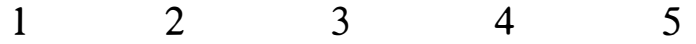

and at / bash will / reveal / the truth / to thee

Se trata de versos en pentámetro yámbico, estructura poética muy utilizada en la antigüedad, especialmente por cierta poesía de la tradición grecolatina. Estrella se enfrenta a un reto mayor que los anteriores: traducir el poema, utilizar palabras similares que hagan resolver el acertijo y tratar de cumplir con la característica métrica del poema original.

Antes de analizar la versión al español, se presenta el significado del poema en cuestión.

\begin{tabular}{|c|c|}
\hline Versión original & Traducción \\
\hline an ancient word of wisdom frees this scroll & una palabra de sabiduría libera este pergamino \\
and helps us keep her scatter $d$ family whole & y nos ayuda a mantener intacta su familia dispersa \\
a headstone praised by templars is the key & la llave es una lápida alabada por templarios \\
and atbash will reveal the truth to thee & y el Atbash ${ }^{14}$ te revelará la verdad \\
\hline
\end{tabular}

El contenido de la estrofa no da lugar a la confusión. Sin embargo, ahora viene lo interesante para el traductor: transmitir ese

14 Atbash: código hebreo de codificación. Brown, El código Da Vinci, 376. 
mensaje en forma de poema lo más semejante al original. A continuación se observa la propuesta de Juanjo Estrella para la versión al español.

Versión al español:

Palabra sabia, antigua, el pergamino abre y mantiene unida a su camada.

Lápida por templarios venerada es la llave, y el Atbash el camino. ${ }^{15}$

Se reproduce otra estrofa de cuatro versos, pero con respecto al uso del pentámetro yámbico Estrella opta por versos endecasílabos, lo cual es común en este tipo de traducciones de poemas y versos, pues esta métrica en cuanto a su forma y cantidad de sílabas se asemeja al pentámetro en los versos en inglés.

$\mathrm{Pa} / \mathrm{la} / \mathrm{bra} / \mathrm{sa} / \mathrm{bia}$, an/ti/gua, el/ per/ga/mi/no (11 sílabas) a/bre y/ man/tie/ne u/ni/da a /su/ ca/ma/da. (11 sílabas) Lá/pi/da/ por/ tem/pla/rios/ ve/ne/ra/da (11 sílabas) es/ la/ lla/ve/, y el /A/tbash/ el/ ca/mi/no. (11 sílabas)

Es de resaltar el trabajo de reescritura poética que tiene que realizar el traductor, puesto que aunque varía la métrica, logra producir una estrofa endecasílaba conocida como cuarteto, y que tiene la característica de tener rima consonante en los versos uno y cuatro, y dos y tres. No obstante tan precioso trabajo, Estrella cae en un error que puede tergiversar la base teórica e histórica de la novela.

Al cambiar de pentámetro yámbico a endecasílabo todo lo que Robert Langdon dice sobre el pentámetro en la versión en inglés queda anulado en la versión es español. Incluso se llega a omitir un párrafo entero de la novela original y que para los estudiosos de la novela sería de valiosa importancia.

15 Brown, El Código Da Vinci, 375. 


\begin{tabular}{|c|c|}
\hline Versión original & Versión de Juan jo Estrella \\
\hline $\begin{array}{l}\text { "Langdon had come across this } \\
\text { meter of ten over the years while } \\
\text { researching secret societies across } \\
\text { Europe, including just last year in } \\
\text { the Vatican Secret Archives. }\end{array}$ & $\begin{array}{l}\text { Langdon se había topado a menu- } \\
\text { do con aquel tipo de pie a lo largo } \\
\text { de sus investigaciones sobre las } \\
\text { sociedades secretas europeas; la } \\
\text { última vez había sido hacía apenas } \\
\text { un año, en los Archivos Secretos } \\
\text { del Vaticano. }\end{array}$ \\
\hline $\begin{array}{l}\text { For centuries, iambic pentameter } \\
\text { had been a preferred poetic meter } \\
\text { of outspoken literati across the glo- } \\
\text { be, from the ancient Greek write } \\
\text { Archilochus to Shakespeare, Mil- } \\
\text { ton, Chaucer, and Voltaire - bold } \\
\text { souls who chose to write their so- } \\
\text { cial commentaries in a meter that } \\
\text { many of the day believe had mys- } \\
\text { tical properties. The roots of iam- } \\
\text { bic pentameterwere deeply pagan. }\end{array}$ & $\begin{array}{l}\text { Durante siglos, el endecasílabo ha- } \\
\text { bía sido el preferido de los literatos } \\
\text { más prominentes de todo el mun- } \\
\text { do, desde Arquíloco, el poeta de la } \\
\text { Grecia clásica, hasta Shakespeare, } \\
\text { Milton, Chaucer y Voltaire, men- } \\
\text { tes preclaras que optaron por verter } \\
\text { sus opiniones sociales en una es- } \\
\text { tructura métrica que para muchos, } \\
\text { en su época, tenía propiedades mís- } \\
\text { ticas y cuyas raíces se hundían en el } \\
\text { mundo pagano. }\end{array}$ \\
\hline $\begin{array}{l}\text { Iambs, two syllables with opposite } \\
\text { emphasis. Stressed and unstres- } \\
\text { sed. Yin yang. A balanced pair. } \\
\text { Arranged in strings of five. Pent } \text { - } \\
\text { meter. Five for the pentacle of Ve- } \\
\text { nus and the sacred famine. }\end{array}$ & \\
\hline $\begin{array}{l}\text { "It's a pentameter!" Teabing blur- } \\
\text { ted, turning to Langdon. "And the } \\
\text { verse is in English! La lingua } \\
\text { pura!" } 16\end{array}$ & $\begin{array}{l}\text { —Es un endecasílabo! —excla- } \\
\text { mó Teabing girándose para mirar a } \\
\text { Langdon. }{ }^{17}\end{array}$ \\
\hline
\end{tabular}

16 Brown, The Da Vinci Code, 329

17 Brown. trad. El Código Da Vinci, 376. 
A pesar del esfuerzo de Estrella, en esta ocasión distorsiona el contenido de la novela y hace al lector pensar y creer algo que no es cierto. Una cosa es el pentámetro yámbico y otra el endecasílabo. En este caso el error no radica exclusivamente en que tradujo con otro tipo de métrica, ya es difícil producir esa estrofa con un contenido que se adaptara a la original. Como se puede observar en este segmento de la novela original, se hace referencia a que el pentámetro yámbico fue utilizado por ilustres literatos y hace referencia a la poesía griega, incluso a Shakespeare. Por su parte, en la versión al español se establece esa misma relación pero con el endecasílabo, lo cual es falso. A pesar de reconocer que la novela es ficción, está basada en algunos datos históricos y reales; en este caso si se cambia esa información se cae en un error. En estas situaciones es donde hace falta una nota del traductor; algo tan sencillo como una aclaración habría salvado esta carencia. Una nota explicando que cuando la versión al español menciona el endecasílabo debería entenderse por pentámetro yámbico, que es la forma original de la estrofa. Y agregar que esto se da por motivos de la traducción del poema al tener como resultado versos endecasílabos.

\section{Poema 2}

A pesar de que el primer verso de esta nueva estrofa se cita en el capítulo 78, el poema completo aparece cuatro capítulos después, en el capítulo 82. Esta nueva estrofa consta de cuatro versos y de nuevo usa el pentámetro yámbico.

Versión original:

In London lies a knight a Pope interred.

His labor's fruit a Holy wrath incurred.

You seek the orb that ought be on his tomb.

It speaks of Rosy flesh and seeded womb. ${ }^{18}$

18 Brown, The Da Vinci Code, 364 
Análisis métrico:

In Lon / don lies / a knight / a Pope / interred.

His la / bor's fruit / a Ho / ly wrath / incurred.

You seek / the orb / that ought / be on / his tomb.

It speaks / of Ro / sy flesh / and seed / ed womb.

Significado del contenido del poema:

\begin{tabular}{|c|c|}
\hline Versión original & Traducción \\
\hline In London lies a knight a Pope interred. & $\begin{array}{c}\text { En Londres reposa un caballero } \\
\text { enterrado por un Papa. } \\
\text { His labor's fruit a Holy wrath incurred. } \\
\text { El fruto de su labor provocó } \\
\text { una ira sagrada. }\end{array}$ \\
$\begin{array}{c}\text { You seek the orb that ought be on } \\
\text { his tomb. }\end{array}$ & $\begin{array}{c}\text { Busca el orbe que debe estar sobre } \\
\text { su tumba. }\end{array}$ \\
$\begin{array}{c}\text { It speaks of Rosy flesh and seeded } \\
\text { Womb. }\end{array}$ & Habla de carne rosa y de vientre \\
encintado.
\end{tabular}

De nueva cuenta aparece una estrofa que tendría que traducirse con un significado y forma similar a la original. Estrella se ve ante un reto parecido al anterior, y en esta ocasión mantendrá la uniformidad del endecasílabo como en el primer poema.

Versión al español:

En la ciudad de Londres, enterrado

por el Papa, reposa un caballero.

Despertaron los frutos de sus obras

las iras de los hombres más sagrados.

El orbe que en su tumba estar debiera

buscad, os hablará de muchas cosas,

de carne rosa y vientre fecundado. ${ }^{19}$

19 Brown. El Código Da Vinci. 416-417. 
El contenido de la versión en español es muy adecuado con el contenido de la versión original. Básicamente tiene el mismo mensaje y eso resalta la capacidad del traductor de manejar este tipo de traducción. No obstante, ya no se mantiene en las cuatro líneas de la estrofa original, sino que le suma tres líneas.

En/ la/ ciu/dad/ de/ Lon/dres/, en/te/rra/do (11 sílabas)

por/ el $/ \mathrm{Pa} / \mathrm{pa} /$, re/po/sa un/ ca/ba/lle/ro. (11 sílabas)

Des/per/ta/ron/ los/ fru/tos/ de/ sus/ o/bras (11 sílabas)

las/ i/ras/ de/ los/ høm/bres/ más/ sa/gra/dos. (11 sílabas)

$\mathrm{El} / \mathrm{or} / \mathrm{be} / \mathrm{que}$ en/ su/ tum/ba es/tar/ de/bie/ra (11 sílabas)

bus/cad/, os/ ha/bla/rá/ de/ mu/chas/ co/sas, (11 sílabas)

$\mathrm{de} / \mathrm{car} / \mathrm{ne} / \mathrm{ro} / \mathrm{sa}$ y/ vien/tre/ fe/cun/da/do. (11 sílabas)

Para descifrar el poema, la palabra Papa funciona como Pope, no en cuanto pontífice sino como apellido, y ahora sí se rescata ese dato al introducir en el capítulo 95 una Nota del Editor, en la que se indica lo anterior refiriéndose a "Alexander Pope": "Pope": "Papa" o "pontífice" en inglés. ${ }^{20}$

\section{Poema 3}

Pese a que los dos primeros versos del último poema aparecen como preámbulo en el capítulo 104, el poema completo se encuentra en el siguiente capítulo de la novela. Como era de esperar, esta nueṿa entrega es otra estrofa de cuatro versos con la misma rima y con métrica yámbica.

Versión original:

The Holy Grail' neath ancient Roslin waits.

The blade and chalice guarding o'er Her gates.

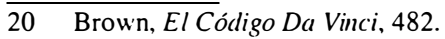


Adorned in masters' loving art, She lies.

She rests at last beneath the starry skies. ${ }^{21}$

Análisis métrico:

The Ho / ly Grail' / neath an / cient Ros / lin waits.

The blade / and cha / lice guar / ding o'er / Her gates.

Adorned / in mas / ters' lo / ving art, / She lies.

She rests / at last / beneath / the star / ry skies.

Significado del poema:

\begin{tabular}{|c|c|}
\hline Versión original & Traducción \\
\hline The Holy Grail' neath ancient & El Santo Grial espera bajo la \\
Roslin waits. & antigua Roslin \\
The blade and chalice guarding o'er & El cáliz y la espada cuidan en sus \\
Her gates. & puertas \\
Adorned in masters' loving art. She lies. & $\begin{array}{c}\text { Reposa adornado con el afable arte } \\
\text { de los maestros } \\
\text { She rests at last beneath the starry skies. }\end{array}$ \\
& $\begin{array}{c}\text { Descansa finalmente bajo el cielo } \\
\text { estrellado }\end{array}$ \\
\hline
\end{tabular}

Este poema cierra el ciclo de acertijos. La estrofa original utiliza la contracción y los apóstrofes para lograr el pentámetro yámbico. Otro aspecto es que en inglés se le otorga género femenino al "Grail", en los últimos dos versos "She lies" y "She rests"; interesante si se toma el contenido de la novela, el cual indica que el Santo Grial realmente se refiere a los restos de María Magdalena, por lo tanto la versión original indica una pista adicional al referirse a She (Ella) como el Grial.

$21 \quad$ Brown. The Da Vinci Code, 482. 
Versión al español:

Bajo la antigua Roslin el Grial

Con impaciencia espera tu llegada.

Custodios y guardianes de sus puertas

Serán por siempre el cáliz y la espada.

Adornada por artes de maestros,

Ella reposa al fin en su morada

$Y$ el manto que la cubre en su descanso

No es otro que la bóveda estrellada. ${ }^{22}$

Como resultado, en lugar de los cuatro versos originales, resultan ocho versos formando una especie de octava.

$\mathrm{Ba} / \mathrm{jo} / \mathrm{la} / \mathrm{an} / \mathrm{ti} / \mathrm{gua} / \mathrm{Ros} / \mathrm{lin} / \mathrm{el} / \mathrm{Grial}$ (10 sílabas) [aguda +1]

Con/ im/pa/cien/cia es/pe/ra/ tu/lle/ga/da. (11 sílabas)

Cus/to/dios/ y /guar/dia/nes/ de/ sus/ puer/tas (11 sílabas)

$\mathrm{Se} / \mathrm{rán} / \mathrm{por} / \mathrm{siem} / \mathrm{pre}$ el/ cá/liz/ y/la es/pa/da. (11 sílabas)

A/dor/na/da/ por/ ar/tes/ de/ ma/es/tros, (11 sílabas)

$\mathrm{E} / \mathrm{lla} / \mathrm{re} / \mathrm{po} / \mathrm{sa} \mathrm{al} / \mathrm{fin} / \mathrm{en} / \mathrm{su} / \mathrm{mo} / \mathrm{ra} / \mathrm{da}$ (11 sílabas)

$\mathrm{Y} \mathrm{el/man/to/} \mathrm{que/} \mathrm{la/} \mathrm{cu/bre} \mathrm{en/} \mathrm{su/} \mathrm{des/can/so} \mathrm{(11} \mathrm{sílabas)}$

No es/ o/tro/ que/ la/ bó/ve/da es/tre/lla/da. (11 sílabas)

Si al poema se le aplica la licencia denominada dialefa en el primer verso con las sílabas 3 y 4 y se separan estas vocales para pronunciarlas en sílabas distintas, entonces se tiene diez sílabas más una sílaba que se suma por terminar en palabra aguda en total el primer verso suma las once sílabas correspondientes.

Volviendo sobre el género, grail es femenino en inglés pero en la versión al español al principio se refiere al éste tal y como es, con género masculino y luego lo cambia al femenino cuando se referir al mismo en los versos 5 (Adornada), 6 (Ella reposa) y 7 (la cubre). Esto 
se pudo haber resuelto si en lugar de Grial se hubiese utilizado un sinónimo femenino como "Copa" o incluso "Sangre" ya que en esta etapa de la novela ya se conoce cual es el significado del Grial.

La estrofa transmite el mensaje en forma acertada y también es de resaltar la rima consonante en los versos 2, 4, 6 y 8; y rima asonante en los versos 1 y 3 , y 5 y 7 . En general se nota un buen trabajo en este último ejemplo a excepción del cambio de género, considerando la dificultad del poema y que además debe mantener una consistencia con respecto a la métrica, pues es casi imposible de igualar la cantidad de versos debido a que el idioma español utiliza mayor cantidad de palabras por lo general son más extensas.

\section{Conclusión}

El trabajo de traducción de Juanjo Estrella nos parece entre regular y aceptable, si se tiene en cuenta que presenta algunos errores. Entre las razones por las cuales se cayó en estos problemas se podrían mencionar muchas causas; el factor tiempo es uno de los principales. En este tipo de obrasliterarias, que tienen una gran demanda, como todo libro de éxito en ventas, la presión por sacar a tiempo una edición en otro idioma agobia al traductor y lo limita hasta cierto punto, dejando de lado aspectos que podría mejorar si tuviera más tiempo para entregar el trabajo.

En cuanto a la reelaboración de criptogramas en español, este proceso de traducción implica mucho conocimiento en cultura general e imaginación, pues pasar de un mensaje codificado a otro similar que incluya de forma oculta otro mensaje resulta muy complejo, sobre todo si se considera que lo que realmente se traduce es una inscripción oculta pero con palabras similares para que tenga sentido con la frase original.

En términos generales el trabajo es bueno y eso también es de rescatar, en especial la creación de los versos en español. No se pone en duda la capacidad de Estrella, pero como toda producción de 
grandes proporciones, es esencial recordar la ayuda de todo el equipo de traductores y colaboradores que apoyaron su trabajo con ideas y sugerencias que dieron como resultado muy buenos poemas.

Algunos dirán que la traducción es adecuada, otros la criticarán mucho; pero cada quien puede tener su propio concepto. El lector común que solo tiene la oportunidad de apreciar la versión en español, quedará satisfecho con el trabajo de traducción y es posible que ni siquiera note los errores que pueda tener la obra. Si es un lector más informado, tendrá algunas dudas al respecto en la forma en cómo habrá sido el mensaje y contenido original de la novela y si le es posible indagará un poco para resolver sus dudas. Pero la única forma de saber si Estrella hizo un buen trabajo es leyendo ambas versiones. En este sentido un número menor de lectores tendrá la habilidad, paciencia y dedicación para hacer esta doble lectura y comparar las obras.

$\mathrm{Al}$ confrontar las obras surgen muchas interrogantes, en cuanto al fondo y la forma de la novela. Este trabajo se centró en la traducción de criptogramas y versos, pero así como se lograron identificar algunas fallas y aciertos sería interesante ahondar mucho más en otros detalles, en especial en cuanto a su contenido.

Quien traduce requiere un pensamiento crítico que lo lleve a preguntarse si el trabajo está bien realizado, debe investigar a fondo cada decisión y analizar las decisiones que vaya a tomar; debe tener una mente abierta y prestar atención a todo lo que lee. ${ }^{23}$ Conviene motivar a quienes traducen para no conformarse con la primera impresión y si notan algo que no encaja, dediquen un poco de su tiempo a investigar, porque una obra literaria (y no solo literaria) puede variar muchísimo de un idioma a otro con solo omitir o cambiar una frase.

Retomando la labor de Juanjo Estrella, su trabajo valió la pena a pesar de los reparos señalados; teniendo en cuenta los factores como tiempo y presión, aparte de ser una versión en el español peninsular y

23 Ingrid Gólcher. "Educación del traductor como pensador crítico", Letras, 36 (2004) 65-80. 
no latinoamericano, el lector final quedaría satisfecho. Si bien aún quedan otras interrogantes como la decisión de emplear un vocabulario que se adapte mejor a la obra o de omitir o pasar por alto detalles de la obra original, esa será labor para otros que deseen investigar por ejemplo, ¿cuál vocablo de cinco letras utilizó para traducir "apple" (palabra que abría el segundo criptex en la novela) y por qué se decidió a usar ese término? 